\title{
Applying engineering feedback analysis tools to climate dynamics
}

\author{
Douglas G. MacMynowski and Eli Tziperman
}

\begin{abstract}
The application of feedback analysis tools from engineering control theory to problems in climate dynamics is discussed through two examples. First, the feedback coupling between the thermohaline circulation and wind-driven circulation in the North Atlantic Ocean is analyzed with a relatively simple model, in order to better understand the coupled system dynamics. The simulation behavior is compared with analysis using root locus (in the linear regime) and describing functions (to predict limit cycle amplitude). The second example does not directly involve feedback, but rather uses simulation-based identification of low-order dynamics to understand parameter sensitivity in a model of El Niño/Southern Oscillation dynamics. The eigenvalue and eigenvector sensitivity can be used both to better understand physics and to tune more complex models. Finally, additional applications are discussed where control tools may be relevant to understand existing feedbacks in the climate system, or even to introduce new ones.
\end{abstract}

\section{INTRODUCTION}

What happens when an aerospace controls engineer asks whether the tools that he is familiar with are useful for analyzing feedback in climate systems? While for the most part (with a few potential exceptions noted in Section 4) we do not have control over the feedback in these systems, feedback analysis is certainly relevant for understanding climate systems. Herein we explore the use of analysis tools from engineering control theory to problems in mesoscale climate dynamics through two examples: the coupling between the thermohaline and wind-driven circulations in the North Atlantic, and parameter sensitivity in a model of ElNiño/Southern Oscillation (ENSO). Further details on these examples can be found in $[1,2]$ respectively; herein we frame this research for a controls audience. In addition to the direct benefit of understanding the physics of these two examples, we hope to motivate further research, and to provide an example of interdisciplinary research that could stimulate thought on the broader applicability of controls tools.

The first example explored herein (Section 2) directly applies feedback analysis tools to understanding the coupling between the thermohaline circulation (THC) in the North Atlantic and the wind-driven circulation (WDC). The THC is the meridionally overturning circulation driven by density gradients, which are caused by poleward thermal and salinity gradients. This current transports large amounts of heat polewards and is thus important to the climate system. Simple

This work was supported by the James McDonnell Foundation.

D. G. MacMynowski is in the Department of Control and Dynamical Systems, California Institute of Technology, Pasadena, CA 91125. macmardgecds. caltech. edu

E. Tziperman is with Earth \& Planetary Sciences, and Division of Engineering \& Applied Sciences, Harvard University, Cambridge, MA 02138. elideps.harvard. edu
THC models are bi-stable, with a bifurcation at sufficient values of fresh-water forcing in the North Atlantic [3,4]. Accurately understanding its dynamics is therefore important to understanding the effects of anthropogenic climate change (e.g. due to melting polar ice sheets). The winds in the North Atlantic also drive a strong clockwise surface ocean circulation, and of course these two systems are coupled. One-way coupling has been explored in simple models [5], while here we look at the two-way feedback coupling. Near the Hopf bifurcation point, stability can be predicted from the linearization, and simple root locus analysis is useful for understanding the influence of the feedback. In the coupled system, a limit cycle develops beyond the bifurcation, and describing functions can be used on a reduced-order model to predict the limit cycle amplitude as a function of feedback gain. Understanding how feedback changes the dynamics is important in understanding the behavior both of more complicated models, and of the real ocean.

The second example explored herein (Section 3) doesn't directly involve feedback, but again uses tools familiar to controls engineers, but not to most climate researchers. Here, we use system identification to extract low-dimensional models from a complex simulation of ENSO in order to understand parameter sensitivity. El-Niño is the largest coherent inter-annual signal in the climate, affecting rainfall and temperature patterns across the globe. It arises from coupled atmosphere/ocean physics in the tropical Pacific ocean $[4,6,7]$ with a dominant period between two and seven years. Eigenvalue variation (or root locus) has been used to explore ENSO dynamics in simple, analytically tractable models $[4,8,9]$, and some limited information obtained in more complex models [10]. Here we extract the desired information from simulations, allowing the eigenvalue and eigenvector parametric sensitivity to be obtained for more complex models that more accurately capture the physics and retain the effects of multiple physical parameters. We demonstrate this approach on the intermediate complexity model of Zebiak and Cane [11]. Further details, and the use of this information to further the understanding of ENSO physics can be found in [2]. It is hoped that the parametric sensitivity will aid in tuning more complex general circulation models (GCMs) of the entire climate system and improve their ability to accurately model ENSO.

Section 4 describes potential future research problems involving analysis of feedbacks in climate phenomena at multiple time-scales, and gives a brief survey on the potential for controlling elements of Earth's climate over a range of scales from weather up to the entire globe. 

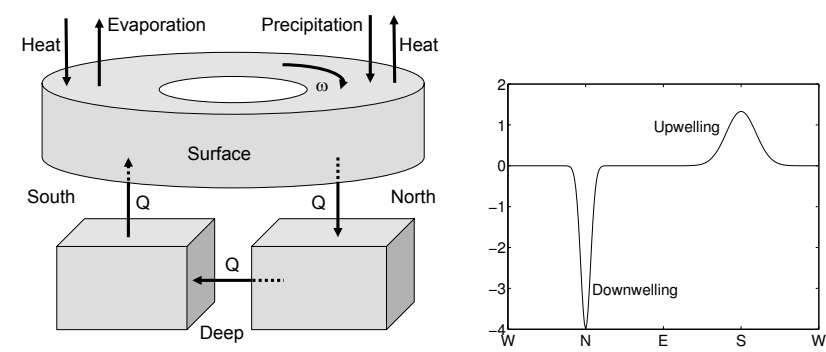

Fig. 1. Schematic of the model for coupled wind-driven and thermohaline circulation, from [5]. The system is forced by differential fresh-water (salinity) forcing $F$ in the northern and southern (mid-latitude) sections. The assumed upwelling/downwelling distribution is also shown.

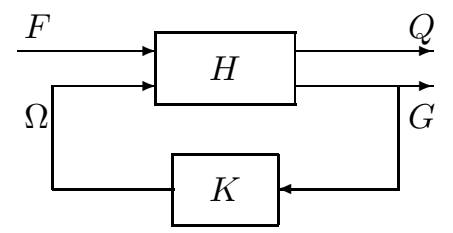

Fig. 2. Block diagram of feedback interaction; $H$ is the model of [5], in which the WDC affects the THC. $K$ is the new feedback we introduce, allowing the WDC $\Omega$ to change as function of the SST gradient $G$.

\section{THERMOHALINE CIRCULATION}

Stommel [3] modeled the THC with "boxes" representing the north and south (equatorial) surface ocean, connected below through the deep ocean, with differential thermal and fresh-water (FW) or salinity forcing. The latter represents differential evaporation/precipitation, as well as the freshwater due to rivers or ice melt in the North Atlantic. For a wide range of FW forcing, the model exhibits two possible stable equilibria: a strong poleward flow (the current climate state) where the thermal effect on density dominates, and a weak reverse flow dominated by the counteracting density effect of salinity. For sufficiently large FW forcing, the thermally dominant branch loses stability and the THC shuts down.

Building on this simple box model, Pasquero and Tziperman [5] developed a model that also includes the effect of the wind-driven circulation: the surface ocean is replaced by an annulus, with boxes for the deep ocean, as shown in Fig. 1. The states are the salinity and temperature around the annulus and in the deep boxes, and the equations are discretized advection/diffusion. The circulation $Q$ advects anomalies, and also depends linearly on the state, and the equations are therefore quadratically nonlinear. The wind driven circulation (WDC) affects the THC by the advection of salinity anomalies into deep water formation sites. In this model, the stable salinity dominant equilibrium disappears, and the thermal equilibrium loses stability at high FW forcing $F$ in a Hopf bifurcation to a limit cycle.

However, the thermohaline circulation also affects the WDC because it affects the meridional atmospheric temperature gradient and therefore the strength of the atmospheric winds. We model this by including a linear feedback between the poleward sea surface temperature (SST) gradient $G$ and the WDC strength $\Omega$ as shown in Fig. 2. Aside from the additional feedback the equations can be found in [5]. For an equilibrium state $x_{0}$ corresponding to WDC strength $\Omega_{0}$,

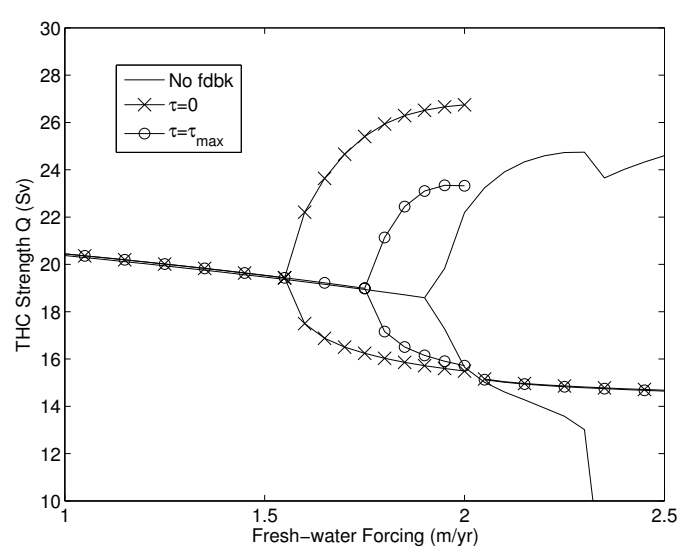

Fig. 3. THC bifurcation behavior as a function of FW forcing with and without feedback between THC and WDC. The behavior depends on the feedback time constant $\tau$. Results are plotted for fixed WDC (solid line, from [5]), for $\tau=0$ (' $\times$ ') and $\tau=\tau_{\max }$ (' $\circ$ '). The feedback is destabilizing for $\mathrm{FW}$ forcing below $1.9 \mathrm{~m} / \mathrm{yr}$. Beyond the bifurcation point the limit cycle max/min amplitude are plotted. For $F>2$, the system with feedback converges to a stable steady state. Units for $Q$ are Sverdrup $\left(10^{6} \mathrm{~m}^{3} / \mathrm{s}\right)$. From [1], (C) copyright 2006 American Meteorological Society.

we assume that the steady state WDC as a function of SST gradient $G=C_{G} x$ is given by

$$
\Omega^{*}(x)=\Omega_{0}\left(1+k \frac{C_{G}\left(x-x_{0}\right)}{C_{G} x_{0}}\right),
$$

with feedback gain $k$, and we assume the WDC equilibration has time constant $\tau$ so that the perturbation in $\Omega$ satisfies

$$
\frac{\mathrm{d} \Omega}{\mathrm{d} t}=\frac{1}{\tau}\left[\Omega^{*}(x)-\Omega\right],
$$

For this model, the feedback can have a significant effect on the dynamics of the coupled system. For a reasonable choice of parameters, the feedback destabilizes the THC equilibrium for moderate fresh-water forcing, leading to an earlier bifurcation and correspondingly larger limit cycle at $\mathrm{FW}$ forcing above the bifurcation point. As the forcing is increased further, the feedback results in a new stable equilibrium instead of the large amplitude limit cycle that develops without feedback. These effects are shown through simulation in Fig. 3.

In the linear region before bifurcation, the dependence of stability on feedback parameters can also be rapidly explored using root locus tools. We linearize about the nofeedback equilibrium at $F=1.9 \mathrm{~m} / \mathrm{yr}$, and compute the transfer function of $H$ from input $\Omega$ to output $G$. Near the bifurcation, the response is close to a $2^{\text {nd }}$-order lightly damped system with phase $-30^{\circ}$ at the peak frequency. Textbook root locus analysis shows that the departure angle of the dominant pole should also be $-30^{\circ}$, plus whatever phase lag is introduced through the feedback $K$ in (2). Thus, the root locus behavior in Fig. 4 can be approximately predicted without resorting to simulation. Furthermore, the shift in bifurcation behavior in Fig. 3 as a function of the WDC equilibration time constant $\tau$ can be understood: if $\tau=0$, then $K$ adds no phase lag, and the poles move rapidly into the right-half plane. We chose $\tau_{\max }=20 \mathrm{yrs}$, 


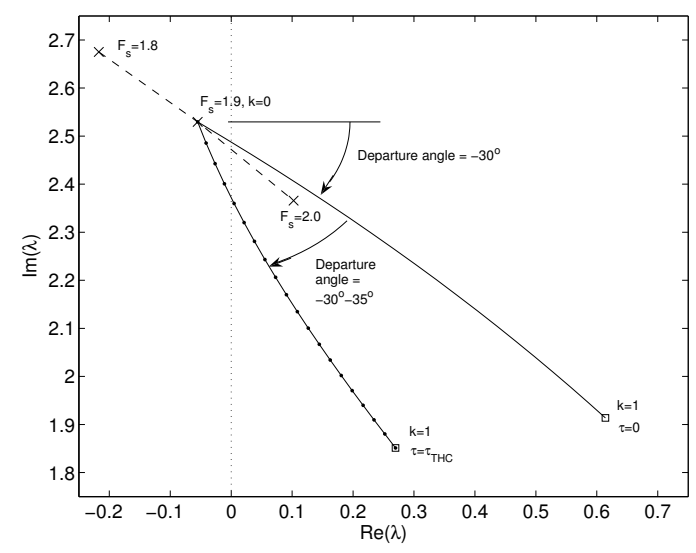

Fig. 4. Root locus of dominant eigenvalue of linearized coupled system at $F=1.9$ for gain $0 \leq k \leq 1$, for time constant $\tau=0$ (solid) and $\tau=\tau_{\max }\left({ }^{\prime} \cdot\right)$. The pole location for varying $F$ is also shown (dashed). From [1], (c) copyright 2006 American Meteorological Society.

slightly less than the time constant of the poles of $H$, so at $\tau=\tau_{\max }, K$ adds $35^{\circ}$ phase lag. The departure angle then increases to $-65^{\circ}$, resulting in a less rapid destabilization with increasing $k$ than if $\tau=0$. Physically this represents that if the delay between a perturbation and the resulting feedback is in phase, the feedback is strongly destabilizing, while if the resulting feedback is $1 / 4$ cycle out of phase, it has little effect on stability.

While simulation is possible for this simple model, basic root locus tools are useful for understanding whether added feedbacks will be relevant, and stabilizing or not, in more complex models. In addition, it helps understand the dynamic rather than simply quasi-static effects of feedback.

At higher FW forcing where the model exhibits limit cycle behavior, describing function analysis [12] can be used with partial success to predict the dependence of limit cycle amplitude on feedback parameters. We first use model reduction for analytical tractability. Basis functions are obtained using proper orthogonal decomposition (POD) [13], using the observability Gramian as the inner-product weighting to guarantee that dynamically-relevant modes are included [14]. Galerkin projection gives the reduced order model

$$
\dot{q}_{i}=\sum_{j=1}^{n} \mathcal{X}_{i j} q_{j}+\sum_{j=1}^{n} \sum_{k=1}^{n} \mathcal{Y}_{i j k} q_{j} q_{k}+\mathcal{Z}_{i}
$$

where the coefficients $\mathcal{X}_{i j}, \mathcal{Y}_{i j k}$ and $\mathcal{Z}_{i}$ are readily obtained from the original equations as inner products [15]. We seek solutions for the reduced-model state $q(t) \in \mathbb{R}^{n}$ of the form

$$
q(t)=\alpha+\beta \sin \omega t+\gamma \cos \omega t+\delta \sin 2 \omega t+\epsilon \cos 2 \omega t,
$$

where the higher harmonics are included because the limit cycle rapidly becomes non-sinusoidal away from the bifurcation point. Substituting into the dynamics (3), nonzero solutions to the harmonic balance equations correspond to limit cycles. The approach works reasonably well at predicting the limit cycle without feedback (Fig. 5), and for small feedback amplitude. For higher feedback amplitude, additional harmonics would be required in (4) to accurately capture the behavior.
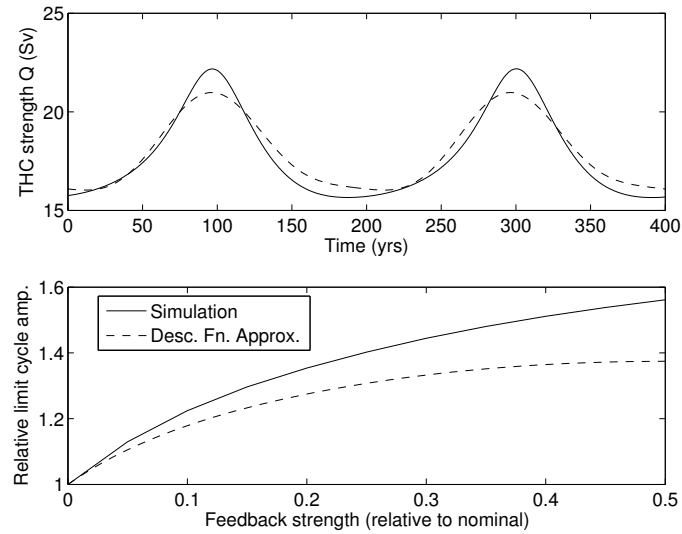

Fig. 5. Describing functions approximation of limit cycle for FW forcing $F_{s}=2.0 \mathrm{~m} / \mathrm{yr}$. Top: without feedback, simulation (solid) and approximation (dashed). Bottom: Trend in limit cycle amplitude with feedback gain for simulation (solid) and describing function analysis (dashed).

\section{EL-NIÑO/SOUTHERN OSCILLATION}

ENSO dynamics arise from feedback coupling between atmosphere and ocean physics in the tropical Pacific [4,6,7], but the impact of ENSO extends over much of the planet. Some models have predicted an increase in ENSO variability as a result of forecast anthropogenic climate changes, others are more ambiguous [16]. In large part, the uncertainty reflects the difficulties in accurately capturing ENSO using General Circulation Models (GCMs) designed to simulate the entire climate [17]. Understanding ENSO dynamics and in particular what parameters determine its period is thus crucial for tuning complex models, and ultimately understanding the effects of climate change on ENSO dynamics.

From a dynamics perspective, ENSO involves primarily a single oscillatory mode $[4,10,18]$. The dynamics are probably stable and damped, driven by weather "noise" external to the system $[10,18,19]$, though possibly unstable, with the observed irregular period resulting from self-sustained chaotic oscillations [20]. The results herein do not depend on knowing which explanation is correct, as we are interested in the underlying linear behavior. In either regime, parameterdependent shifts in the period or damping of the dominant eigenvalues of the linearization give corresponding shifts in the observed spectrum (although this is not rigourously provable in the chaotic case). Previous researchers have explored ENSO eigenvalue variation, but only in simple models $[4,8,9]$, or with a limited set of parameters in more complex models [10]. Here, we extract eigenvalue and eigenvector dependence for many parameters, from a higher-dimensional model. In contrast to the THC example previously, this application does not directly involve feedback analysis, but does use system identification concepts familiar to a controls researcher, but not familiar to a typical climate researcher.

The fundamental elements of the physics are well understood ( [6] is written for a reader with a non-climatology background). A hierarchy of modeling tools have been applied, including numerical fitting of the observed timesequence [18], inverse modeling [21], very low-order physically-motivated oscillator models $[22,23]$, intermediate- 
complexity coupled atmosphere/ocean models [11] and modified general circulation models (GCMs) [24].

The oscillation is amplified by positive Bjerknes feedback [25] between the ocean and atmosphere: increased East-West SST gradient leads to increased atmospheric convection, a strengthened Walker circulation, and increased westward equatorial surface winds that drive the ocean currents and in turn the SST and thermocline slope. The atmospheric adjustment time is rapid, hence this feedback is nearly in phase with a perturbation, and primarily influences the stability, not the period of oscillation. The period is strongly influenced by lags in the dynamic response of the ocean, including both wave dynamics and SST-adjustment time (e.g. [9]).

A single ENSO cycle proceeds as follows. A positive perturbation in the east Pacific SST (for example) decreases the atmospheric winds as described above. The changed wind stress leads to an eastward-propagating equatorial ocean Kelvin wave that deepens the thermocline, and a westwardpropagating off-equatorial Rossby wave that shallows the thermocline depth. The Kelvin wave amplifies the original SST perturbation by changing the temperature of upwelling in the eastern Pacific, and the original anomaly thus grows. Meanwhile, the more slowly traveling Rossby waves reflect off the western Pacific boundary as Kelvin waves. Once these reach the eastern Pacific, they begin to cancel the anomaly there, although it can take some time to accumulate a sufficient cancelling signal, depending on the relative amplitude between the eastern anomaly and the opposite sign returning wave [2,26,27].

We use the Zebiak and Cane (ZC) [11] intermediatecomplexity atmosphere/ocean anomaly model. This has been successfully used for prediction of ENSO [28], and has been the basis for substantial research. Plausible time series and predictions can be obtained either with parameters chosen so that the system exhibits self-sustained chaotic oscillations, or for which the system is stable and forced by stochastic noise. A shallow-water model is used for the ocean and atmosphere, with an embedded ocean-surface mixed-layer, parameterized atmospheric heating due to SST-dependent evaporation and convergence, and parameterized subsurface ocean temperature. The model coarsely discretizes these coupled partial differential equations over the tropical Pacific ocean, leading to $\sim 33000$ state variables. However, the ENSO oscillation itself is a low-dimensional phenomena involving only a few degrees of freedom. Simpler models $[22,23]$ have the advantage of fewer degrees of freedom, and thus improved analytical tractability. These simpler models, however, do not capture the independent effects of multiple parameters. The sensitivity analysis described below uses a simulation-based approach to identify and extract relevant low-dimension information (eigenvalues and eigenvectors) about the system linearization without explicitly constructing the linearization.

The seasonal cycle results in a time-periodic model $x_{k+1}=f\left(x_{k}, j\right)$ where $j=\bmod (k, 12)$ is the month and $x$ the state vector. The annual model $x_{k}=g\left(x_{k-12}\right)$ is time-

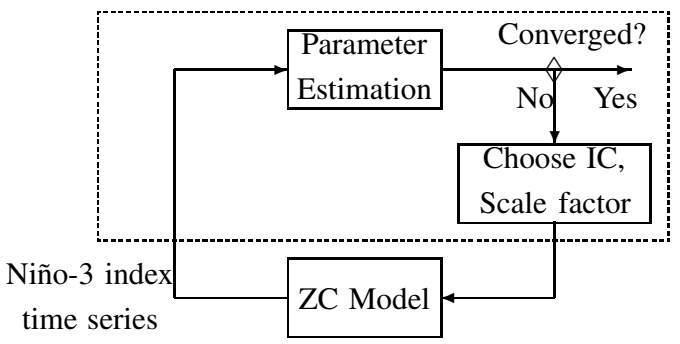

Fig. 6. Schematic of algorithm. The original simulation code ("ZC Model") is used with only minor modification. The outer loop of code chooses appropriate inputs for the simulation to estimate linear model parameters and exits based on a convergence test.

invariant (using Floquet analysis as in [10,29]). An autoregressive (AR)-model

$$
y_{k}=\sum_{i=1}^{N} a_{i} y_{k-12 i}
$$

can be fit to the model output time series $y$ from an unforced simulation from an initial condition close to the dominant eigenvector. (Note that all the data are still used, as the eigenvalues and hence coefficients $a_{i}$ do not depend on the month $j$.) We use $y$ as the scalar Niño-3 index (average SST over a region of eastern Pacific) since the relevant mode will clearly be observable in this output. A recursive stochastic (Kalman filter) formulation to estimate AR-parameters $\hat{a}$ allows small bursts of simulations to be used and convergence monitored. To extract the linearized behavior from the simulation, one code modification is required to scale the state vector at each time step, so $x_{k+1}=\alpha f\left(x_{k}, j\right)$. The only effect of this on the linearization is to add or subtract damping. This allows the damping of the dominant eigenvalue pair to be chosen to be stable but lightly damped, thereby maximizing the quality of system identification on the output time series. The eigenvalues $\lambda$ of the linearization of the modified system are related to those of the original system by the scale factor;

$$
\lambda\left(\frac{\partial(\alpha f)}{\partial x}\right)=\alpha \cdot \lambda\left(\frac{\partial f}{\partial x}\right)
$$

An outer loop can then be written that chooses the simulation initial conditions and scale factor, uses a short burst of simulation to update AR model estimates $\hat{a}$, and iterates until convergence is reached; this is shown schematically in Fig. 6. This approach allows legacy code to be used as a black box.

The eigenvectors corresponding to the dominant complex eigenvalue pair can also be extracted, since in the linearized regime, the response after sufficient time is dominated by this complex eigenvector pair. Given several cycles of statevector history $x_{k}$ then

$$
v=\frac{1}{m} \sum_{k=1}^{m} \lambda^{-k} x_{k}
$$

is a good estimate of the eigenvector. The change in eigenvector as parameters change is also useful to understanding the relevant physics and feedback processes involved. 


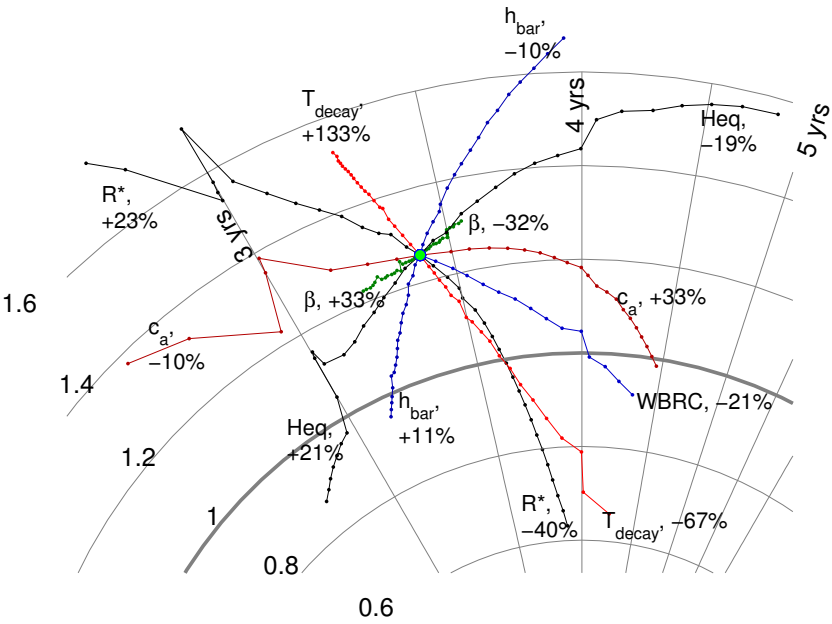

Fig. 7. Eigenvalue sensitivity near nominal ZC parameters, illustrating dependence on ocean/atmosphere coupling $\left(R^{*}\right)$, atmospheric heating parameterization $(\alpha, \beta)$, mean eastern thermocline depth $\left(h_{\text {bar }}\right)$ ocean decay time $\left(T_{\text {decay }}\right)$, atmospheric wave speed $\left(c_{a}\right)$, western boundary reflection coefficient (WBRC) and ocean equivalent depth (Heq). The annual growth rate (lines of constant radii, 0.6 to 1.6) and period (lines of constant phase, 3-5 years) is indicated. Some phase-locking to the seasonal cycle is evident at periods of 3 and 4 years.

Fig 7 shows a typical root locus output for nominal (unstable) parameters in the discrete-time ZC model. Lines of constant radii correspond to equal growth rate, while lines of constant phase correspond to equal period or frequency. Thus the model period is most sensitive to $c_{a}, H_{\mathrm{eq}}$, WBRC, and $h_{\text {bar }}$ with the latter two parameters strongly affecting the damping as well. Together with the shift in the eigenvector, this information leads to insights into the model physics, described more fully in [2]. The parameters $c_{a}$ and $H_{\text {eq }}$ affect the Rossby radius of deformation for the atmosphere and ocean respectively, and their ratio affects which ocean Rossby modes are excited [27,30]. Since higher-order modes travel more slowly, this affects the average wave propagation speed and therefore the delay before cancelling effects reach the eastern Pacific. The other two parameters noted above, WBRC and $h_{\text {bar }}$ don't affect the wave propagation speed, but change the relative amplitude between the growing anomaly in the eastern Pacific, and the strength of the returning reflected wave of the opposite sign. If the anomaly grows more rapidly, then it takes a greater accumulation of returning waves to cancel the perturbation and begin the next cycle of oscillation.

The understanding obtained herein regarding which parameters are relevant in determining ENSO's period, and why, is useful in tuning more complex models. This, in turn, will improve the prediction of the effect on ENSO due to future changes in the climate system.

\section{CONCLUSIONS AND FUTURE WORK}

The two examples described herein illustrate different opportunities for the application of ideas and tools from engineering control theory to research problems in climate dynamics. The first category of opportunities is thus to explore feedbacks within the climate system, while the second category is the use of modeling and model reduction tools.

Both of the problems discussed herein have clear and useful extensions. The THC and WDC coupling can be considered in more complex models to understand whether similar behavior still exists. Additional tools can also be applied to the understanding of ENSO. Formal model reduction tools to extract the dynamically relevant states could be useful [14], both for this and for more complex models. Controllability and observability analysis would be useful for understanding the spatial and temporal sensitivity of ENSO to forcing, and the relevant spatial and temporal information to sense in order to better estimate future evolution.

In addition to THC and ENSO, there are other dynamic phenomena relevant to understanding the climate. Variability occurs on a wide range of time scales, due to the complex nonlinear interactions between the oceans, atmosphere, sea ice, land ice sheets, as well as the land and ocean biospheres. Examples include Dansgaard-Oeschger oscillations (1500yrs) [31], Heinrich events (7000yrs) [32] and global glacial cycles $(100,000 y r s)$ [33]. Despite the tremendous complexity and richness of temporal and spatial scales, in almost all of these examples, the relevant dynamics may be explained in terms of a simple mechanism, often surprisingly well represented by simple idealized models, e.g. $[34,35]$. While progress has been made in understanding these subsystems in isolation, feedback analysis tools could be broadly useful in understanding the dynamics of the interaction between phenomena at multiple time scales. Understanding the characteristics of feedback is also relevant to understanding the probability distribution of global climate change prediction [36].

In addition to the analysis problems described herein, there are several potential areas where human engineering has been suggested to intentionally modify the climate system. The concept of such control has a long history; the initial investment by the U.S. Office of Naval Reseach in the development of numerical weather prediction was substantially motivated by the hope of weather control [37]. Control of weather generally [38] and hurricane track or strength [39,40] have been suggested. However, the dynamics are both chaotic and high dimensional. The former is in principle an advantage, as it leads to high sensitivity, however the inherent uncertainty may make robust control strategies challenging! The studies to date have demonstrated the possibility of control in the absence of model uncertainty, and much more work would need to be done in both controllability analysis and robust feedback design.

Also relevant in any discussion of control is energy input. In this context, it has been shown that a significant shift of human power generation to wind energy would result in a measurable effect on atmospheric winds and in turn on the climate [41]. Thus, contrary to popular wisdom, this scale of energy extraction does have the potential to influence the climate, because the energy mediated through heat and moisture transport is much larger than the direct change in kinetic energy, and thus provides some leverage.

Control of chaotic ENSO dynamics has been described 
[42] although the primary goal here was the demonstration of chaos-control techniques. Nonetheless, ENSO may provide an interesting example for exploring feedback control within climate systems, in part because the important dynamics are relatively low order, making them at least theoretically amenable to modification because predictability is relatively good [28]. This is essential for robustness of any modelbased feedback algorithm.

On a larger scale, geoengineering has been suggested as a possible element of a climate change strategy [43, 44] (see also the editorial section of Climatic Change, Vol. 77, Aug. 2006, pp. 211-248); while most of the current research has focused on the actuation and the environmental risk, there are certainly control aspects to this problem.

Nonetheless, despite the fascination with control of the climate, we expect the most significant benefit to arise from communication between control and climate researchers to be in the analysis of existing feedbacks within the climate system, not in the creation of new feedbacks.

\section{REFERENCES}

[1] D. G. MacMynowski and E. Tziperman, "Two-way feedback interaction between the thermohaline and wind-driven circulations," J. Phys. Oceanogr., vol. 36, no. 5, pp. 914-929, 2006.

[2] - "Factors affecting ENSO's period," in press, J. Atmospheric Sciences, 2008.

[3] H. Stommel, "Thermohaline convection with two stable regimes of flow," Tellus, vol. 13, pp. 224-230, 1961.

[4] H. A. Dijkstra, Nonlinear physical oceanography. Kluwer Academic Publishers, 2000.

[5] C. Pasquero and E. Tziperman, "Effects of a wind-driven gyre on thermohaline circulation variability," J. Phys. Oceanogr., vol. 34, no. 4, pp. 805-816, Apr. 2004.

[6] H. A. Dijkstra and G. Burgers, "Fluid dynamics of El-Niño variability," Annual Review of Fluid Mechanics, vol. 34, pp. 531-558, 2002.

[7] J. D. Neelin, D. S. Battisti, A. C. Hirst, F.-F. Jin, Y. Wakata, T. Yamagata, and S. Zebiak, "ENSO theory," Special Joint issue of J. Geophys. Res. Atmospheres and J. Geophys. Res. Oceans, vol. 103, no. C7, pp. 14,261-14,290, 1998.

[8] F.-F. Jin and D. Neelin, "Models of interannual tropical oceanatmosphere interaction - a unified view. Part I: numerical results," J. Atmos. Sci., vol. 50, pp. 3477-3503, 1993.

[9] J. D. Neelin, M. Latif, and F.-F. Jin, "Dynamics of coupled oceanatmosphere models: The tropical problem," Ann. Rev. Fluid Mech., vol. 26, pp. 617-659, 1994.

[10] C. J. Thompson and D. S. Battisti, "A linear stochastic dynamical model of ENSO. Part I: Model development," Journal of Climate, vol. 13, pp. 2818-2883, 2000.

[11] S. E. Zebiak and M. A. Cane, "A model El Niño-Southern Oscillation," Mon. Weath. Rev., vol. 115, pp. 2262-2278, 1987.

[12] A. Gelb and W. Vander Velde, Multiple-input Describing Functions and Nonlinear System Design. McGraw Hill, 1968.

[13] J. L. Lumley, Stochastic Tools in Turbulence. Academic Press, 1970.

[14] C. W. Rowley, "Model reduction for fluids, using balanced proper orthogonal decomposition," Int. J. on Bifurcation and Chaos, vol. 15, no. 3, pp. 997-1013, 2005.

[15] C. Rowley, T. Colonius, and R. M. Murray, "Model reduction for compressible flows using POD and Galerkin projection," Physica D, vol. 189 , no. 1-2, pp. 115-129, 2004.

[16] M. A. Cane, "The evolution of El Niño, past and future," Earth and Planetary Science Letters, vol. 230, pp. 227-240, 2005.

[17] K. AchutaRao and K. R. Sperber, "ENSO simulation in coupled oceanatmosphere models: are the current models better?" Climate Dynamics, vol. 27, pp. 1-15, 2006.

[18] G. Burgers, "The El Niño stochastic oscillator," Climate Dynamics, vol. 15, no. 7, pp. 521-531, July 1999 .

[19] S. G. Philander and A. Federov, "Is El Niño sporadic or cyclic?" Annual Rev. Earth Planet. Sci., vol. 31, pp. 579-594, 2003.
[20] E. Tziperman, M. A. Cane, and S. E. Zebiak, "Irregularity and locking to the seasonal cycle in an ENSO prediction model as explained by the quasi-periodicity route to chaos," J. Atmos. Sci., vol. 52, no. 3, pp. 293-306, feb 11995.

[21] C. Penland and P. D. Sardeshmukh, "The optimal-growth of tropical sea-surface temperature anomalies," J. Climate, vol. 8, no. 8, pp. 1999_ 2024, Aug. 1995

[22] M. J. Suarez and P. S. Schopf, "A delayed action oscillator for ENSO,' J. Atmos. Sci., vol. 45, pp. 3283-7, 1988.

[23] F.-F. Jin, "An equatorial ocean recharge paradigm for ENSO. Part I: conceptual model," J. Atmos. Sci., vol. 54, pp. 811-829, 1997.

[24] E. Roeckner, J. M. Oberhuber, A. Bacher, M. Christoph, and I. Kirchner, "ENSO variability and atmosphere response in a global atmosphere-ocean GCM," Climate Dynamics, vol. 12, pp. 737-754, 1996.

[25] J. Bjerknes, "Atmospheric teleconnections from the equatorial Pacific," Mon. Weath. Rev., vol. 97, pp. 163-172, 1969.

[26] M. A. Cane, M. Münnich, and S. E. Zebiak, "A Study of Self-Excited Oscillations of the Tropical Ocean-Atmosphere System. Part I: Linear Analysis,” J. Atmos. Sci., vol. 47, no. 13, pp. 1562-1577, 1990.

[27] B. P. Kirtman, "Oceanic Rossby wave dynamics and the ENSO period in a coupled model," J. Climate, vol. 10, no. 7, pp. 1690-1704, 1997.

[28] D. Chen, M. A. Cane, A. Kaplan, S. E. Zebiak, and D. J. Huang, "Predictability of El Niño over the past 148 years," Nature, vol. 428, pp. 733-736, 2004

[29] F.-F. Jin, D. Neelin, and M. Ghil, "El Niño southern oscillation and the annual cycle: subharmonic frequency-locking and aperiodicity," Physica D, vol. 98, pp. 442-465, 1996.

[30] P. C. F. Van der vaart, H. A. Dijkstra, and F. F. Jin, "The Pacific cold tongue and the ENSO mode: A unified theory within the ZebiakCane model," Journal of the Atmospheric Sciences, vol. 57, no. 7, pp. 967-988, 2000.

[31] GRIP Ice Core Project Members, "Climate instability during the last interglacial period recorded in the GRIP ice core," Nature, vol. 364 , pp. 203-207, 1993.

[32] H. Heinrich, "Origin and consequences of cyclic ice rafting in the Northeast Atlantic Ocean during the past 130,000 years," Quat. Res., vol. 29, pp. 142-152, 1988.

[33] J. R. Petit, J. Jouzel, D. Raynaud, N. I. Barkov, J. M. Barnola, I. Basile, M. Bender, J. Chappellaz, M. Davis, G. Delaygue, M. Delmotte, V. M. Kotlyakov, M. Legrand, V. Y. Lipenkov, C. Lorius, L. Pepin, C. Ritz, E. Saltzman, and M. Stievenard, "Climate and atmospheric history of the past 420,000 years from the Vostok ice core, Antarctica," Nature, vol. 399, pp. 429-436, 1999.

[34] H. Gildor and E. Tziperman, "Sea ice as the glacial cycles climate switch: role of seasonal and orbital forcing," Paleoceanography, vol. 15 , pp. 605-615, 2000.

[35] R. Sayag, E. Tziperman, and M. Ghil, "Rapid switch-like sea ice growth and land ice-sea ice hysteresis," Paleoceanography, vol. 19, no. PA1021, doi:10.1029/2003PA000946, March 2004.

[36] G. H. Roe and M. B. Baker, "Why is climate sensitivity so unpredictable?" Science, vol. 318, pp. 629-632, 2007.

[37] K. Harper, "Research from the boundary layer: Civilian leadership, military funding and the development of numerical weather prediction," Social Studies of Science, vol. 33, no. 5, pp. 667-696, 2003.

[38] R. N. Hoffman, "Controlling the global weather," AMS Bulletin, vol. 83, no. 2, pp. 241-248, Feb. 2002.

[39] —_, "Controlling hurricanes," Scientific American, vol. 291, no. 4, pp. $68-75,2004$

[40] J. M. Henderson, R. N. Hoffman, S. M. Leidner, T. Nehrkorn, and C. Grassotti, "A 4D-Var study on the potential of weather control and exigent weather forecasting," Quarterly J. Royal Met. Soc., vol. 131, pp. 3037-3051, 2005

[41] D. W. Keith, J. F. DeCarolis, D. C. Denkenberger, D. H. Lenschow, S. L. Malyshev, S. Pacala, and P. J. Rasch, "The influence of largescale wind power on global climate," PNAS, vol. 101, no. 46, pp. 16115-16120, 2004.

[42] E. Tziperman, H. Scher, S. E. Zebiak, and M. A. Cane, "Controlling spatiotemporal chaos in a realistic El Niño prediction model," Physical Review Letters, vol. 79, no. 6, pp. 1034-1037, 1997.

[43] S. H. Schneider, "Geoengineering: Could or should we do it," Climatic Change, vol. 33, pp. 291-302, 1996.

[44] D. Keith, "Geoengineering the climate: History and prospect," Annual Rev. Energy Environ., vol. 25, pp. 245-284, 2000. 